\title{
Características nutricionales y fermentativas de ensilados de pasto Camerún con plátano Pelipita ${ }^{1}$
}

\section{Nutritional and fermentative characteristics of Cameroon grass silage with Pelipita banana}

\author{
Michael López-Herrera², Augusto Rojas-Bourrillon², Carlos Zumbado-Ramírez ${ }^{3}$
}

\begin{abstract}
Resumen
El objetivo de esta investigación fue determinar las características nutricionales y fermentativas de los ensilados de pasto Camerún con sustitución por fruto inmaduro de plátano Pelipita (Musa ABB). El experimento se desarrolló entre mayo y setiembre de 2015, se utilizó diseño irrestricto al azar con cuatro tratamientos y tres niveles de sustitución con fruto $(15,30$ y $45 \%$ peso/peso) y un control ( $0 \%$ de fruto) con melaza (3\% peso/peso). Cada tratamiento tuvo cinco repeticiones. Los materiales fueron cosechados y picados en Upala, Costa Rica; la parte experimental se desarrolló en Montes de Oca, Costa Rica. Las mezclas se almacenaron durante sesenta días en bolsas de $5 \mathrm{~kg}$. Los tratamientos con fruto inmaduro presentaron valores de $\mathrm{pH}$ y nitrógeno amoniacal mayores que los tratamientos con melaza, también tuvieron concentraciones mayores de carbohidratos no fibrosos y energía. La materia seca solo aumentó en el tratamiento con 45\% de sustitución. Sin embargo, en todos los tratamientos donde se utilizó el fruto se redujo la concentración de los componentes de la fibra y la digestibilidad de la fibra detergente neutro, esto debido a un incremento en la concentración de lignina. Los materiales ensilados tuvieron el potencial para producir 1,50-1,70 $\mathrm{kg}$ leche/vaca, al consumir $5 \mathrm{~kg}$ material verde (MV)/animal, y de 4,40-5,20 kg/vaca leche, al consumir $15 \mathrm{~kg} \mathrm{MV} /$ animal, con base en el requerimiento de energía neta de lactancia con una tasa de pasaje por consumo tres veces mayor que el mantenimiento (3x).
\end{abstract}

Palabras clave: conservación de forrajes, Cenchrus purpureus, forrajes tropicales, aditivos para ensilaje, ensilaje.

\begin{abstract}
The objective of this research was to determine the nutritional and fermentative characteristics of Cameroon grass silage with replacement of immature Pelipita banana fruit (Musa ABB). The experiment was carried out between May and September of 2015, using a random and unrestricted design with four treatments and three substitution levels of fruit $(15 ; 30,45 \% \mathrm{w} / \mathrm{w})$ and one control ( $0 \%$ fruit) with molasses $(3 \% \mathrm{w} / \mathrm{w})$. Each treatment had five replicates. The materials were harvested and minced in Upala, Costa Rica; the experimental part was developed in Montes de Oca, Costa Rica. The blends were stored for sixty days in $5 \mathrm{~kg}$ bags. Treatments that used immature fruit presented higher
\end{abstract}

1 Recibido: 27 de julio, 2016. Aceptado: 14 de diciembre, 2016. Este trabajo forma parte del Proyecto de la Vicerrectoría de Investigación de la Universidad de Costa Rica 739-B4-115, San José, Costa Rica.

2 Universidad de Costa Rica, Escuela de Zootecnia, Centro de Investigación en Nutrición Animal. Montes de Oca, San José, Costa Rica. michael.lopez@ucr.ac.cr, augusto.rojas@ucr.ac.cr

3 Ministerio de Agricultura y Ganadería, Agencia de Servicios Agropecuarios. Upala, San Carlos, Alajuela, Costa Rica. zumbadocarlos@gmail.com 
values of $\mathrm{pH}$ and ammonia nitrogen, than treatments that used molasses, treatments that used immature fruit also showed higher concentrations of non-fibrous carbohydrates and energy. Dry matter only increased in the treatment that had a $45 \%$ substitution. However, in all treatments where the fruit was used, the concentration of the fiber components and the digestibility of the neutral detergent fiber were reduced, due to an increase in lignin concentration. The ensiled materials had the potential to produce $1.50-1.70 \mathrm{~kg}$ milk/cow, when are consumed $5 \mathrm{~kg}$ fresh material (MV)/animal, and 4.40-5.20 kg milk/cow when consume $15 \mathrm{~kg}$ MV/animal, based on the requirement of net breastfeeding energy with a consumption rate three times higher than maintenance $(3 \mathrm{x})$.

Keywords: forage preservation, Cenchrus purpureus, tropical forages, silage additives, silage.

\section{Introducción}

La productividad de los sistemas de rumiantes en condiciones tropicales, está asociada a la calidad de los recursos forrajeros con que cuenta el productor, estos recursos a su vez pueden ser afectados por diferentes factores, tales como: la radiación solar, la precipitación, el tipo de suelo y el manejo que reciben por parte de los productores.

Como mecanismo de defensa y fortaleza a las condiciones climáticas en la región tropical, los forrajes tropicales poseen alto contenido de fibra y reducida digestibilidad (Sampaio et al., 2009), lo que representa una limitante en la productividad de los sistemas de producción de rumiantes; esto se debe a que existe una restricción en el consumo voluntario de los forrajes, como consecuencia del alto contenido de fibra detergente neutro (FDN) y una disminución en la digestibilidad como resultado del contenido de lignina en la planta (Cruz-Calvo y Sánchez, 2000; Moore y Jung, 2001). La combinación de estos factores afecta el rendimiento de los animales, debido a que no es posible llenar los requerimientos diarios de otros nutrimentos, como la energía.

De acuerdo con varios autores, los pastos tropicales de la especie Cenchrus purpureus, son materiales forrajeros que permiten el manejo en sistemas de corte y acarreo, además tienen una producción de biomasa alta, lo que ha generalizado su uso en los sistemas de producción de rumiantes del trópico, ya sea como fuente de alimento fresco, recién cortado o como forraje conservado mediante la técnica de ensilaje (Muia et al., 2000; Araya-Mora y Boschini-Figueroa, 2005). Sin embargo, son materiales con bajo contenido de energía y proteína, junto a un alto contenido de fibra, situación que limita su uso en los sistemas de rumiantes.

El ensilaje es una técnica de conservación de forraje por vía húmeda, que consiste en almacenar forrajes en estado verde en ausencia de oxígeno, donde ocurren transformaciones químicas y físicas que definen su calidad (Hiriart, 2008). Existen diferentes tipos de materiales que pueden ser utilizados como aditivos para mejorar los procesos químicos del ensilaje o la calidad final del material conservado; un ejemplo de ello son los subproductos agroindustriales con alto contenido de nutrimentos (Gutiérrez et al., 2003; López-Herrera et al., 2016).

El uso de musáceas en la alimentación de rumiantes está ampliamente estudiado (Mohapatra et al., 2010), diversos estudios indican del uso de los frutos maduros o verdes, las cáscaras y el pseudotallo de la planta (Happi-Emaga et al., 2008; Katongole et al., 2008; Archimède et al., 2010; 2012). El uso de frutos de musáceas en combinación con los forrajes tropicales, permite incrementar el contenido de energía en las dietas de animales rumiantes en producción (Archimède et al., 2010), además, debido a su alto contenido de almidones (Mohapatra et al., 2010), el uso de los frutos puede resultar en un incremento en la productividad, ya que puede afectar la producción de ácidos orgánicos en el rumen (France y Dijkstra, 2005).

El objetivo de esta investigación fue evaluar las características de fermentación y nutricionales de los ensilados de mezclas de pasto Camerún (Cenchrus purpureus) con diferentes niveles de inclusión de fruto inmaduro de plátano Pelipita (Musa ABB). 


\section{Materiales y métodos}

Para el desarrollo del experimento se utilizaron frutos de plátano Pelipita (Musa acuminata x balbisiana, Grupo ABB) y pasto Cenchrus purpureus cv Camerún, cosechado a 55 días, cuya composición nutricional se indica en el Cuadro 1. El pasto y los materiales utilizados fueron obtenidos en la finca agroecológica Siempreverde ubicada en Upala, Alajuela, Costa Rica; esta zona posee una humedad relativa promedio de $84 \%$, con un promedio de precipitación de $2500 \mathrm{~mm} /$ año y una temperatura promedio de $25^{\circ} \mathrm{C}$ (IMN, 2017). Los frutos de plátano Pelipita que fueron utilizados estaban completamente llenos y engrosados, aunque presentaban una coloración verde.

Cuadro 1. Composición nutricional del plátano Pelipita (Musa acuminata x balbisiana, Grupo ABB) y pasto Cenchrus purpureus cv Camerún, cosechado a 55 días, utilizados para la preparación de ensilados. San José, Costa Rica. 2016.

Table 1. Nutritional composition of Pelipita plantain (Musa acuminata x balbisiana, ABB Group) and Cenchrus purpureus cv. Cameroon, harvested at 55 days, used for silage preparation. San Jose, Costa Rica. 2016.

\begin{tabular}{lcc}
\hline & Plátano Pelipita & Pasto Camerún \\
\hline MS (\%) & 28,01 & 20,71 \\
PC (\% MS) & 3,90 & 7,11 \\
FDN (\% MS) & 8,90 & 72,95 \\
FDA (\% MS) & 5,70 & 45,01 \\
Lignina (\% MS) & - & 2,87 \\
CNF (\% MS) & 80,7 & 8,11 \\
NDT (\%) & 87,2 & 51,2 \\
\hline
\end{tabular}

MS: materia seca; PC: proteína cruda; FDN: fibra en detergente neutro; FDA: fibra en detergente ácido; CNF: carbohidratos no fibrosos; NDT: nutrientes digestibles totales / DM: dry matter; CP: crude protein; NDF: neutral detergent fiber; ADF: acid detergent fiber; NFC: non-fibrous carbohydrates; TDN: total digestible nutrient.

Para la conservación del material se utilizaron bolsas de polietileno verdes para ensilaje vacío, con capacidad para $50 \mathrm{~kg}$ y con un grosor de $0,0063 \mathrm{~mm}$. Cada bolsa fue considerada como una unidad experimental. Todos los materiales fueron picados con una picadora eléctrica hasta obtener un tamaño de partícula entre 3-5 cm. El experimento constó de un diseño irrestricto al azar completamente aleatorizado, aplicando tres niveles de sustitución de pasto Camerún por fruto de plátano Pelipita $(15,30,45 \%$ peso/peso) e inóculo bacterial (1 1/t), preparado fermentando suero de leche y microorganismos. El tratamiento control fue elaborado con melaza (3\% peso/peso) e inóculo bacterial artesanal $(1 \mathrm{l} / \mathrm{t})$, con respecto al pasto. Cada tratamiento fue repetido cinco veces para un total de veinte bolsas.

De cada tratamiento se tomaron muestras previo al proceso de ensilaje, estas fueron llevadas al laboratorio de bromatología del Centro de Investigaciones en Nutrición Animal (CINA) para analizar su composición. El resto del material fue embolsado para someterlo al proceso de ensilaje, para tal fin, se utilizó una bomba de succión para extraer el aire de las bolsas y favorecer el proceso anaeróbico. Los silos se mantuvieron en condiciones ambientales (temperatura de $25{ }^{\circ} \mathrm{C}$ y humedad relativa $>80 \%$ ) por sesenta días. Posterior a este periodo, se abrieron las bolsas y se muestreó con una sonda de PVC al interior de la bolsa; la muestra fue analizada en el laboratorio. A todas las muestras se les realizaron los análisis de materia seca (MS), proteína cruda (PC), extracto etéreo (EE) y cenizas de 
acuerdo a la AOAC (1998); carbohidratos no fibrosos (CNF) según la metodología de NRC (2001); concentración de fibra en detergente neutro (FDN), fibra en detergente ácido (FDA), hemicelulosa (HEM) y lignina, siguiendo las metodologías descritas por Mertens (2002). La concentración de FDN indigestible fue estimada a partir del contenido de lignina de acuerdo a la ecuación [1] descrita en el trabajo de Van-Soest et al. (2005).

$\mathrm{FND}_{\mathrm{i}}=$ Lignina $\times 2,4$

Para la determinación de las características fermentativas se realizó la medición del potencial de hidrógeno $(\mathrm{pH})$ con un potenciómetro con electrodo de hidrógeno, también se midió el nitrógeno amoniacal mediante la metodología descrita por Tobía (2004).

Se estimó el contenido de nutrimentos digestibles totales (NDT) en los materiales ensilados con las ecuaciones descritas por Weiss (2004), y para estimar el fraccionamiento de la energía se utilizaron las ecuaciones del NRC (2001). Además, se determinó la proteína insoluble en FDN (PIDN) y la proteína insoluble en FDA (PIDA), de acuerdo con los procedimientos descritos en Licitra et al. (1996).

Se calculó el aporte (Mcal/día) de energía neta de lactación $\left(\mathrm{EN}_{\mathrm{L}}\right)$ de cada una de las mezclas ensiladas, con base en tres niveles de consumo de material verde fresco (MV) (5, 10 y $15 \mathrm{~kg} \mathrm{MV} / \mathrm{vaca} / \mathrm{día}$ ), dichos aportes fueron comparados con el aporte energético equivalente que haría un ensilado de maíz. Finalmente, los contenidos de energía se cotejaron contra los requerimientos para la producción de leche de una vaca de $454 \mathrm{~kg}$ en lactancia media, cuya leche posee $3,0 \%$ de proteína verdadera y $4 \%$ de grasa (NRC, 2001).

Para el análisis de la información, se realizó un análisis de varianza utilizando el programa estadístico INFOSTAT (Di-Rienzo et al., 2016), de acuerdo a la siguiente ecuación:

$\mathrm{y}_{\mathrm{ij}}=\mu+\mathrm{P}_{\mathrm{i}}+\varepsilon_{\mathrm{ij}}$

Donde:

$\mathrm{y}_{\mathrm{ij}}$ : es la variable de respuesta obtenida de la ecuación.

$\mu$ : es la media general.

$\mathrm{P}_{\mathrm{i}}$ : es el efecto i ésimo de la inclusión de plátano Pelipita.

$\varepsilon_{\mathrm{ij}}$ : término de error, donde $\varepsilon \sim \mathrm{N}\left(0, \sigma^{2}\right)$.

Cuando se determinó la significancia de los efectos principales, la comparación entre medias se realizó mediante la prueba de Tukey con un nivel de significancia de 0,05 .

\section{Resultados y discusión}

\section{Parámetros de fermentación de los ensilados}

El potencial de hidrógeno $(\mathrm{pH})$ del tratamiento que presentó mayor grado de acidificación fue donde se incorporó melaza, el mismo fue significativamente diferente $(\mathrm{p}<0,0001)$ de los tratamientos con plátano Pelipita, aunque no se encontraron diferencias entre los tratamientos con adición del plátano (Cuadro 2). Estas diferencias pueden ser debidas a una menor concentración de carbohidratos solubles en el forraje y en el fruto del plátano, en combinación al elevado contenido de humedad $(<18 \%$ MS) en la mezcla forrajera a ensilar. 
Cuadro 2. Valores de $\mathrm{pH}$, nitrógeno amoniacal $\left(\mathrm{NH}_{3} / \mathrm{NT}\right)$ y materia seca $(\mathrm{MS})$ de los ensilados de pasto camerún (Cenchrus purpureus) con la inclusión de fruto inmaduro de plátano pelipita (Musa ABB), posteriores a sesenta días de fermentación. San José, Costa Rica. 2016.

Table 2. Values of $\mathrm{pH}$, ammonia nitrogen $\left(\mathrm{NH}_{3} / \mathrm{NT}\right)$ and dry matter (DM) of Cameroon grass (Cenchrus purpureus) with immature Pelipita plantain fruit (Musa ABB) silage, after 60 days of fermentation. San Jose, Costa Rica. 2016.

\begin{tabular}{lccc}
\hline Tratamientos & $\mathbf{p H}$ & $\mathbf{N H}_{3}(\% \mathbf{N T})$ & $\mathbf{M S}(\%)$ \\
\hline pasto camerún+melaza & $3,7 \pm 0,03^{\mathrm{a}}$ & $3,0 \pm 0,5^{\mathrm{a}}$ & $17,6 \pm 0,3^{\mathrm{b}}$ \\
pasto camerún+15\% plátano pelipita & $4,6 \pm 0,3^{\mathrm{b}}$ & $6,0 \pm 2,1^{\mathrm{b}}$ & $16,9 \pm 0,2^{\mathrm{a}}$ \\
pasto camerún+30\% plátano pelipita & $4,8 \pm 0,1^{\mathrm{b}}$ & $8,0 \pm 2,9^{\mathrm{b}}$ & $17,9 \pm 0,5^{\mathrm{ab}}$ \\
pasto camerún+45\% plátano pelipita & $4,8 \pm 0,1^{\mathrm{b}}$ & $6,3 \pm 1,1^{\mathrm{b}}$ & $18,5 \pm 0,2^{\mathrm{c}}$ \\
\hline Valor de p & $<0,0001$ & 0,0073 & $<0,0001$ \\
\hline
\end{tabular}

Letras distintas en la misma columna son diferentes $(\mathrm{p}<0,05)$. NT: nitrógeno total; MS: materia seca / Different letters in the same column are different $(\mathrm{p}<0.05)$. TN: total nitrogen; DM: dry matter.

Los materiales altos en humedad ( $<18 \% \mathrm{MS}$ ), favorecieron los procesos de fermentación secundaria, sobretodo la fermentación de los clostridios que degradan el ácido láctico (Hiriart, 2008). Para conservar forrajes altos en humedad, se debe agregar más cantidad de carbohidratos solubles que permitan reducir el potencial de hidrógeno a cuatro o menos (Kung y Shaver, 2001), y de esta manera obtener un ensilado estable y con pocas pérdidas de nutrimentos.

Los valores de $\mathrm{pH}$ de los tratamientos con inclusión de guineo cuadrado fueron más elevados que los del tratamiento con melaza, por lo que, se asume que el plátano Pelipita no poseía una cantidad suficiente de carbohidratos solubles que permitiera reducir el valor de $\mathrm{pH}$ en el ensilado, es decir que, aunque el fruto posee alrededor de $80,1 \%$ de carbohidratos no fibrosos (Cuadro 1), estos podrían estar en una forma poco aprovechable por las bacterias en el silo. En los frutos inmaduros, los carbohidratos dominantes están en forma de almidones y muy pocos azúcares (Barrera et al., 2010), los cuales tienen poca participación en el proceso de ensilaje, debido a que las enzimas amilolíticas bacterianas presentan poca o nula actividad en medios ácidos, como los que se desarrollan durante el ensilaje (Neureiter, 2005).

En otras investigaciones, donde se utilizaron fuentes ricas en almidones como aditivos para la conservación de forrajes, se encontró que el 90-100\% del almidón de cebada y avena fue recuperado posterior al ensilaje (Yitbarek y Tamir, 2014). Sin embargo, en ensilajes de leguminosas es posible utilizar maíz como aditivo, mientras que la cantidad de leguminosa sea menor al 30\% de la mezcla (Mülhbach, 2001); posterior a esto, se debe agregar una fuente alta en azúcares solubles como la melaza (Yitbarek y Tamir, 2014), esto indica que podría existir un efecto de acuerdo con el tipo de almidón presente en el aditivo y el tipo de forraje utilizado.

Los valores de $\mathrm{pH}$ de los tratamientos con inclusión de plátano Pelipita fueron menores que los valores obtenidos en ensilados de pasto elefante con diferentes tipos de aditivos (5,9-6,5) (Cavali et al., 2010). Estas diferencias pueden surgir a partir de un alto contenido de humedad en las mezclas ensiladas, una reducida concentración de carbohidratos solubles en los tratamientos con fruto de plátano, comparado con el tratamiento donde se utilizó melaza, debido a procesos de fermentación heterofermentativos y clostridiales (McDonald, 1981).

De manera complementaria, se determinó la concentración de nitrógeno amoniacal; en este caso se encontró que los tratamientos donde se utilizó plátano como fuente de carbohidratos, no fueron diferentes entre sí, pero diferentes $(\mathrm{p}<0,05)$ al tratamiento con melaza, ya que este último presentó menor concentración de nitrógeno amoniacal (Cuadro 2). El nitrógeno amoniacal puede ser afectado por un exceso en el contenido de proteína cruda, 
la concentración de carbohidratos solubles, la capacidad amortiguadora del forraje, el exceso de humedad en el silo o la mala compactación del material (Hiriart, 2008).

Los valores de concentración de nitrógeno amoniacal obtenidos en los tratamientos con inclusión de plátano estuvieron correlacionados $(\mathrm{p}=0,79)$ con los valores de $\mathrm{pH}$, de esta manera, conforme aumentó el valor de $\mathrm{pH}$, así mismo aumentó la concentración de nitrógeno amoniacal. Este comportamiento se debió a que un ambiente de menor acidez ( $\mathrm{pH}$ cercano a 5) aumenta la proliferación de microorganismos proteolíticos que descomponen las proteínas en amonio y aminas, lo que resulta en un fuerte olor a orina o amoniaco, situación que reduce el valor nutricional del material ensilado y podría afectar negativamente el consumo voluntario de los animales (Hiriart, 2008). Sin embargo, para clasificar un ensilado como de buena calidad, la concentración de nitrógeno amoniacal máxima debe ser de 7\% del nitrógeno total (Kung y Shaver, 2001), esto muestra que los valores obtenidos en esta investigación provienen de un adecuado proceso de fermentación, a pesar del elevado contenido de humedad en el silo.

Los valores de nitrógeno amoniacal determinados en esta investigación, fueron similares de los tratamientos con inclusión de plátano Pelipita obtenidos por Cubero et al. (2010) en ensilados de maíz (6,01 - 7,00\% $\mathrm{NH}_{3} / \mathrm{NT}$ ); mientras que el tratamiento con melaza presentó valores menores. Todos los tratamientos evaluados presentaron niveles de nitrógeno amoniacal inferiores a los reportados por Zanine et al. (2010) (14,6-21,9\% $\left.\mathrm{NH}_{3} / \mathrm{NT}\right)$. El menor valor obtenido con la inclusión de melaza se atribuye a que esta última provee una mayor cantidad de nutrimentos, principalmente carbohidratos solubles, con respecto al fruto de plátano Pelipita, lo que redujo la degradación de los compuestos nitrogenados de las mezclas ensiladas, además, los frutos pueden tener diferentes concentraciones de azúcares o almidones, lo que puede afectar el resultado del ensilaje (Happi-Emaga et al., 2008).

El contenido de MS de los tratamientos se vio favorecido de manera significativa $(\mathrm{p}<0,05)$ por la inclusión de plátano (Cuadro 2). De esta manera, el tratamiento con mayor contenido de materia seca fue el tratamiento con mayor inclusión de plátano, mientras que el de menor contenido de materia seca fue el de 15\% de inclusión del fruto. Esto se debe a que, el fruto de plátano posee alrededor de $28 \%$ de materia seca (Cuadro 1), lo que incrementó el contenido de nutrimentos presentes en la mezcla.

Aunque la inclusión de plátano Pelipita aumentó el contenido de nutrimentos en las mezclas ensiladas, la cantidad de materia seca es insuficiente para asegurar un adecuado ambiente para las bacterias productoras de ácido láctico, al contrario, este tipo de ambiente es ideal para la proliferación de bacterias del género Clostidium (Kung y Shaver, 2001; Hiriart, 2008), que podrían afectar de manera negativa el proceso de ensilaje y aumentar la concentración de nitrógeno, amoniacal en el silo. Sin embargo, en este caso, el incremento en el valor de nitrógeno amoniacal no respondió al contenido de humedad, ya que no hubo diferencia en la concentración de $\mathrm{NH}_{3}$, aunque aumentó el contenido de materia seca, lo que hizo suponer que existió un efecto del menor contenido de carbohidratos solubles en el silo.

El contenido de MS en todos los tratamientos fue menor que el obtenido por Betancourt $(2004)(19,18 \%)$ en ensilados de maíz, aunque mayores a los reportados en el trabajo de Cubero et al. (2010) (14,63 - 15,71\%), también con ensilados de maíz. Los resultados de los ensilados analizados también resultaron ser menores a los obtenidos por Maza et al. (2011) (19,0 - 24,0\%) en ensilados de pasto Pennisetum sp. con yuca fresca. Estas diferencias pueden estar determinadas por la especie forrajera y las diferencias climáticas al momento de elaboración de los ensilados (Sánchez y Soto, 1998; López-Herrera y Briceño-Arguedas, 2016), lo que reafirma la necesidad de aumentar el contenido de materia seca de los forrajes.

\section{Componentes celulares de los forrajes ensilados}

El contenido de proteína cruda de los ensilados fue influenciado por la inclusión de plátano, de esta manera, conforme se realizó mayor inclusión del fruto, así disminuyó el contenido de esta fracción en el ensilado (Cuadro 3). El 
Cuadro 3. Concentraciones de proteína cruda (PC), extracto etéreo (EE), cenizas y carbohidratos no fibrosos (CNF) de los ensilados de pasto Camerún (Cenchrus purpureus) con la inclusión de fruto inmaduro de plátano Pelipita (Musa ABB), posteriores a 60 días de fermentación. San José, Costa Rica. 2016.

Table 3. Concentrations of crude protein (CP), ether extract (EE), ash, and non-fibrous carbohydrates (NFC) of Cameroon grass (Cenchrus purpureus) with immature Pelipita plantain fruit (Musa ABB) silage, after 60 days of fermentation. San José, Costa Rica. 2016.

\begin{tabular}{lcccc}
\hline Tratamientos & PC $(\% \mathbf{M S})$ & EE $(\%$ MS) & Cenizas $(\%$ MS) & CNF $(\%$ MS) \\
\hline pasto Camerún+melaza & $7,0 \pm 0,4^{\mathrm{c}}$ & $5,7 \pm 0,9^{\mathrm{b}}$ & $9,9 \pm 0,7^{\mathrm{c}}$ & $5,3 \pm 2,1^{\mathrm{a}}$ \\
pasto Camerún+15\% plátano Pelipita & $5,6 \pm 0,6^{\mathrm{b}}$ & $2,9 \pm 0,8^{\mathrm{a}}$ & $7,6 \pm 0,8^{\mathrm{b}}$ & $18,5 \pm 5,9^{\mathrm{b}}$ \\
pasto Camerún+30\% plátano Pelipita & $5,2 \pm 0,3^{\mathrm{b}}$ & $2,2 \pm 0,6^{\mathrm{a}}$ & $8,2 \pm 0,7^{\mathrm{b}}$ & $25,5 \pm 4,8^{\mathrm{c}}$ \\
pasto Camerún+45\% plátano Pelipita & $4,7 \pm 0,15^{\mathrm{a}}$ & $2,3 \pm 0,4^{\mathrm{a}}$ & $6,7 \pm 0,5^{\mathrm{a}}$ & $33,9 \pm 3,9^{\mathrm{d}}$ \\
Valor de p & $<0,0001$ & $<0,0001$ & $<0,0001$ & $<0,0001$ \\
\hline
\end{tabular}

Letras distintas en la misma columna son diferentes $(\mathrm{p}<0,05)$. PC: proteína cruda; EE: extracto etéreo; CNF: carbohidratos no fibrosos / Different letters in the same column are different $(\mathrm{p}<0.05)$. CP: crude protein; EE: ether extract; NFC: non fibrous carbohydrates.

contenido de proteína del plátano Pelipita fue bajo (3,90\% MS), lo que provocó que al aumentar su inclusión se redujera la concentración de proteína en el ensilado, por lo tanto, el tratamiento que presentó mayor contenido de proteína fue el control sin fruto, mientras que el de menor concentración de proteína cruda fue el de 45\% de adición de plátano.

Esta disminución en el valor de proteína cruda podría provocar una deficiencia en el metabolismo del nitrógeno, sobre todo si estos ensilados se utilizan en gran cantidad dentro de una ración, ya que reducirían el valor de proteína cruda de la dieta, lo que compromete el funcionamiento del rumen (Calsamiglia et al., 2010). Dietas con concentraciones de proteína cruda menores a 6-8\%, pueden afectar de manera negativa el metabolismo del nitrógeno en rumen y el consumo de alimento (Van-Soest, 1994), lo que hace necesario complementar con materiales altos en proteína cruda o con fuentes de nitrógeno no proteico como aditivos al ensilaje (Yitbarek y Tamir, 2014).

Los ensilados con inclusión de plátano Pelipita, contenían niveles de proteína cruda menores a los reportados para ensilados de maíz y sorgo (6,1 y 6,7\% MS, respectivamente) (Abdelhadi y Santini, 2006), también fueron menores a las concentraciones encontradas por Jensen et al. (2005) (8,4-8,8\% MS) en ensilados de maíz con diferentes estados de madurez del forraje; esto se debe a un menor contenido de esta fracción en el fruto conforme aumenta la inclusión (Cuadro 1). Finalmente, los valores obtenidos en esta investigación se encuentran en el rango de proteína encontrado en ensilados de pasto Pennisetum sp. con melaza a diferentes edades de corte $(4,93-7,10 \%$ MS) (Aganga et al., 2005); sin embargo, se debe reforzar la dieta con este nutrimento, sobre todo si se utilizan niveles altos de fruto en la mezcla a ensilar.

La inclusión de fruto de plátano aumentó la concentración de proteínas ligadas a la fibra detergente neutro y a la fibra detergente ácido. En los ensilados, los valores de PIDN resultaron hasta 3,5 veces mayores, con respecto al tratamiento con uso de melaza; mientras que, en el caso de la PIDA el incremento llegó hasta siete veces más que el tratamiento con uso de melaza. Esto puede ser debido a la concentración de las proteínas ligadas a la fibra en el fruto, sin embargo, no se realizó un análisis al respecto, en esta investigación, por lo que se requiere profundizar más sobre este comportamiento para definir si existe algún efecto.

En cuanto al extracto etéreo de los ensilados, su concentración se redujo con la inclusión del fruto en contraposición con el ensilado elaborado con melaza $(\mathrm{P}<0,05)$, aunque no se encontraron diferencias entre los tratamientos con inclusión de fruto (Cuadro 3). Las diferencias derivadas de la inclusión del plátano en el ensilaje se pudieron deber al bajo contenido de grasa en el fruto (0,36\%) (Espitia-Pérez et al., 2013). Los valores de extracto etéreo obtenidos en este estudio fueron similares a los encontrados en ensilados de pasto Pennisetum 
sp. con adición de yuca (2,07-2,22\% MS) (Maza et al., 2011). El tratamiento con melaza mostró un contenido extracto etéreo mayor (5,69\%), al reportado en pasto Cenchrus purpureus (1,29-1,41\% MS) (Chacón-Hernández y Vargas-Rodríguez, 2009), pero similar a los encontrados por Lounglawan et al. (2014) (3,5-6,3\% MS), debido posiblemente a condiciones de manejo y edad del pasto.

Las cenizas fueron afectadas de manera significativa por la inclusión de plátano Pelipita, de esta manera, donde la concentración de cenizas fue menor conforme se incrementó el nivel de fruto en la mezcla ensilada (Cuadro 3). Al igual que en los otros componentes celulares, la concentración de cenizas disminuyó, producto de la baja concentración de esta fracción $(5,01 \pm 0,06 \%$ MS) en el fruto del plátano.

Los ensilados obtenidos en este estudio poseían una concentración aceptable de cenizas, lo que coincide con lo reportado por Hoffman (2005) quien señaló que el contenido de cenizas en ensilados de pastos y leguminosas varía alrededor del 9,0\% MS. Los valores obtenidos en todos los ensilados, fueron mayores a los obtenidos en diversos estudios (5,61-5,81\% MS) (Cubero et al., 2010) (3,2-4,3\% MS) (Jensen et al., 2005), con ensilados de maíz. El tratamiento en el que se usó melaza presentó valores menores a los obtenidos en diferentes cultivares de pasto Pennisetum purpurem (15,1-21,4\% MS) (Araya-Mora y Boschini-Figueroa, 2005). Además, fue menor a la concentración obtenida por Egbunuogie y Bamikole (2014) en ensilados de pasto Pennisetum purpurem secado al sol y conservado por diez semanas (13,5\% MS). En este caso, las diferencias pudieron ser provocadas por la desigualdad en la edad de cosecha (Lounglawan et al., 2014), o a procesos adicionales aplicados al forraje, como el secado (López-Herrera et al., 2014).

La concentración de carbohidratos no fibrosos se vio influenciada $(\mathrm{p}<0,05)$ por la inclusión del plátano, de esta manera, conforme hubo mayor inclusión del fruto, aumentó el contenido de esta fracción en el ensilado (Cuadro 3). El tratamiento que tuvo el menor contenido de carbohidratos no fibrosos fue el que contenía melaza (5,28\% MS), mientras que los tratamientos con inclusión de plátano Pelipita presentaron un aumento lineal en el contenido de esta fracción, donde la mayor concentración fue la obtenida en el tratamiento con $45 \%$ de inclusión de fruto $(33,98 \% \mathrm{MS})$. De acuerdo con lo anterior, se esperaría un mejor proceso fermentativo en los tratamientos con mayor concentración de carbohidratos no fibrosos, esto debido a que se estaría incorporando mayor cantidad de azúcares, almidones, pectinas y ácidos orgánicos a la mezcla (NRC, 2001). Sin embargo, es posible que la mayor parte de los carbohidratos no fibrosos agregados fueran almidones que, tal y como se citó anteriormente, no participan del proceso fermentativo cuando provienen de frutos verdes de plátanos u otras musáceas (Barrera et al., 2010).

Los valores de carbohidratos no fibrosos en los tratamientos ensilados fueron mayores a los obtenidos en los materiales frescos $(0,64-1,25$ veces mayores), esto debido a que, durante el proceso de ensilaje se produjeron gran cantidad de ácidos orgánicos, producto del metabolismo de las bacterias durante la fermentación del forraje (Muck et al., 2007), mismos que son incluidos como parte de los carbohidratos no fibrosos al momento de la estimación (NRC, 2001). Los valores obtenidos en los materiales ensilados con inclusión de fruto de plátano Pelipita fueron mayores a los publicados por Cubero et al. (2010) (17,1-25,6\% MS) con ensilados de maíz, mientras que el tratamiento con adición de melaza presentó valores menores. Los pastos Cenchrus purpureus, poseen bajas concentraciones de carbohidratos no fibrosos (Manyawu et al., 2003; Bureenok et al., 2012), lo que obliga al uso de aditivos altos en carbohidratos solubles para asegurar su adecuada conservación (Yang et al., 2006; Yitbarek y Tamir, 2014). Se debe realizar más investigación para conocer el aporte de carbohidratos solubles del plátano Pelipita al proceso de ensilaje.

\section{Componentes de la pared celular en los ensilados}

Los componentes de la pared celular de los materiales ensilados fueron influenciados por la inclusión de fruto de plátano Pelipita $(\mathrm{p}<0,05)$, ya que conforme aumentó el contenido de fruto en la mezcla, se redujo la concentración de fibra en detergente neutro (Cuadro 4). El tratamiento con mayor concentración de fibra en detergente neutro fue 
el que poseía melaza (64,18\% MS); esto se debió a que la concentración de fibra en el pasto es muy alta (Manyawu et al., 2003; Bureenok et al., 2012), mientras que la concentración del fruto es muy baja (Cuadro 1), por este motivo, conforme aumentó su cantidad en la mezcla se redujo la concentración de fibra (Cuadro 4).

Cuadro 4. Concentraciones de los componentes de la pared celular de los ensilados de pasto Camerún (Cenchrus purpureus), con la inclusión de fruto inmaduro de plátano Pelipita (Musa ABB), posteriores a 60 días de fermentación. San José, Costa Rica. 2016.

Table 4. Concentrations of silage cell wall components of Cameroon grass (Cenchrus purpureus) with immature Pelipita plantain fruit (Musa ABB) silage, after 60 days of fermentation. San Jose, Costa Rica. 2016.

\begin{tabular}{|c|c|c|c|c|c|c|}
\hline Tratamientos & $\begin{array}{c}\text { FDN } \\
(\% \text { MS })\end{array}$ & $\begin{array}{c}\text { FDA } \\
(\% \text { MS })\end{array}$ & $\begin{array}{c}\text { HEM } \\
(\% \text { MS })\end{array}$ & $\begin{array}{c}\text { LIG } \\
(\% \text { MS })\end{array}$ & $\begin{array}{c}\text { PIDN } \\
(\% \text { PC) }\end{array}$ & $\begin{array}{c}\text { PIDA } \\
(\% \text { PC) }\end{array}$ \\
\hline pasto Camerún + melaza & $72,9 \pm 2,8^{\mathrm{d}}$ & $46,4 \pm 2,7^{\mathrm{c}}$ & $26,5 \pm 0,5^{\mathrm{b}}$ & $4,9 \pm 0,8^{\mathrm{a}}$ & $12,5 \pm 7,2^{\mathrm{a}}$ & $7,6 \pm 5,1^{\mathrm{a}}$ \\
\hline pasto Camerún $+15 \%$ plátano Pelipita & $67,8 \pm 4,5^{\mathrm{c}}$ & $43,5 \pm 2,4^{\mathrm{c}}$ & $24,3 \pm 2,3^{\mathrm{b}}$ & $6,0 \pm 0,4^{\mathrm{b}}$ & $42,5 \pm 7,7^{\mathrm{b}}$ & $27,2 \pm 6,7^{\mathrm{b}}$ \\
\hline pasto Camerún $+30 \%$ plátano Pelipita & $60,9 \pm 4,5^{b}$ & $39,2 \pm 2,7^{\mathrm{b}}$ & $21,8 \pm 2,0^{\mathrm{a}}$ & $6,3 \pm 0,3^{\mathrm{b}}$ & $37,6 \pm 6,7^{b}$ & $31,1 \pm 2,8^{\mathrm{b}}$ \\
\hline pasto Camerún $+45 \%$ plátano Pelipita & $54,3 \pm 3,3^{\mathrm{a}}$ & $34,2 \pm 2,5^{\mathrm{a}}$ & $20,1 \pm 1,2^{\mathrm{a}}$ & $6,5 \pm 0,3^{\mathrm{b}}$ & $44,4 \pm 6,6^{\mathrm{b}}$ & $32,2 \pm 7,1^{\mathrm{b}}$ \\
\hline Valor de $\mathrm{p}$ & $<0,0001$ & $<0,0001$ & 0,0001 & 0,0003 & $<0,0001$ & $<0,0001$ \\
\hline
\end{tabular}

Fibra en detergente neutro (FDN), fibra en detergente ácido (FDA), hemicelulosa (HEM), lignina (LIG), proteína insoluble en detergente neutro (PIDN), proteína insoluble en detergente ácido (PIDA) / Neutral detergent fiber (NDF), acid detergent fiber (FDA), hemicellulose (HEM), lignin (LIG), neutral detergent insoluble protein (NDIP), acid detergent insoluble protein (ADIP).

Letras distintas en la misma columna son diferentes $(\mathrm{p}<0,05) /$ Different letters in the same column are different $(\mathrm{p}<0.05)$.

Cuando se utiliza la ecuación descrita por López-Herrera et al. (2014), donde se relaciona la FDN con consumo de MS, los tratamientos ensilados oscilaron entre 1,86 y 2,68\% del peso corporal, además, todos los tratamientos con inclusión de plátano presentaron un potencial de consumo de materia seca comparable al que poseen los ensilados de maíz descritos en los trabajos de Betancourt (2004) y Cubero et al. (2010) (57,1-66,5\% FDN), ya que permiten un consumo alrededor de 1,80-2,10\% del peso vivo del animal. Se debe considerar que los ensilados obtenidos no pueden ser utilizados como única fuente de forraje, debido a su bajo contenido de proteína (Cuadro 3), por lo que, se deben utilizar como un complemento dentro de una ración balanceada. Sin embargo, con esta comparación se puede apreciar el efecto de la fibra en detergente neutro sobre el consumo de alimento (Lu et al., 2005) y cómo el tratamiento con menor inclusión del fruto, es el que propicia un mayor llenado físico del rumen.

Los valores de fibra en detergente neutro obtenidos variaron entre 44,7-64,2\% MS, así, todos los tratamientos, excepto el de mayor inclusión del fruto, fueron similares a los obtenidos por Ward et al. (2001) $(54,5-63,6 \%$ MS) con ensilados de sorgo forrajero, maíz y mijo, y a los reportados por Abdelhadi y Santini (2006) con ensilados de maíz (50,9\% MS). Además, todos los tratamientos fueron menores a los valores publicados en un estudio con ensilados de pasto Cenchrus purpureus con diferentes aditivos (62,3-72,6\% MS) (Bureenok et al., 2012). Las diferencias encontradas en el contenido de fibra en detergente neutro en este estudio y otros similares indican que, todos los tratamientos se pueden catalogar como ensilados de buena calidad y permiten un buen consumo cuando se utilizan en dietas para animales rumiantes.

El contenido de fibra en detergente neutro indigestible fue mayor conforme aumentó la inclusión del fruto de plátano $(\mathrm{p}>0,05)$; el tratamiento con inclusión de melaza fue el que presentó el menor valor de esta fracción; y se diferenció $(\mathrm{p}<0,05)$ de los demás. También se encontró que los tratamientos en fresco y los ensilados fueron significativamente diferentes $(\mathrm{p}<0,05)$. 
Los valores de fibra en detergente neutro indigestible fluctuaron entre 21,53-38,33\% de la FDN total para los tratamientos en fresco, y entre 16,08-28,70\% de la FDN total para los tratamientos ensilados. Estos valores concuerdan con los resultados obtenidos por Detmann et al. (2004) quienes indicaron que los valores promedio de fibra en detergente neutro indigestible de varios géneros de pastos tropicales varió entre 15-45\% de la FDN total.

Los valores de fibra en detergente neutro indigestible aumentaron de manera proporcional a la inclusión de plátano Pelipita en la mezcla ensilada (Figura 1). Este incremento en el contenido de fibra indigestible obedeció a un incremento en la concentración de lignina en las mezclas forrajeras, debido a que para la estimación de esta fracción se utiliza el valor de la concentración de lignina en el forraje (Van-Soest et al., 2005). La lignina aumentó en la mezcla forrajera debido a que la concentración de este compuesto fue mayor en las cáscaras del fruto, con respecto al contenido de lignina del pasto (Cuadro 1). El valor promedio de lignina en las cáscaras de los frutos varió entre 6-16,8\% MS (Happi-Emaga et al., 2008; Mohapatra et al., 2010). Este incremento en el contenido de lignina es contraproducente, ya que reduce la digestibilidad del forraje (Grabber 2005; Ramírez et al., 2002), en este caso redujo la digestibilidad de la fibra consumida por el animal, lo que disminuyó el valor alimenticio del forraje.

La fibra de los tratamientos ensilados resultó más digestible que la de los tratamientos frescos (Figura 1). Estas diferencias se originaron debido a que los ensilados poseían una menor concentración de fibra en detergente neutro, ya que durante el proceso de ensilaje se consumió una parte de la hemicelulosa y la celulosa del forraje (Thomas et al., 2013). En esta investigación se encontró una disminución en el contenido de hemicelulosa (entre 18,7 y $30,2 \%$ ) y celulosa (entre 1,7 y $12,2 \%$ ) de los forrajes, al pasar de fresco a ensilado, lo que coincide con la afirmación anterior.

La concentración de fibra en detergente ácido fue influenciada por la inclusión de plátano Pelipita en la mezcla, al igual que con la fibra en detergente neutro, el tratamiento con menor concentración de fibra en detergente ácido fue el que contenía $45 \%$ de fruto de plátano $(34,21 \%$ MS), esto podría indicar una mayor digestibilidad, ya que niveles de fibra en detergente ácido altos se relacionan con niveles de digestibilidad bajos (Ramírez et al., 2002).

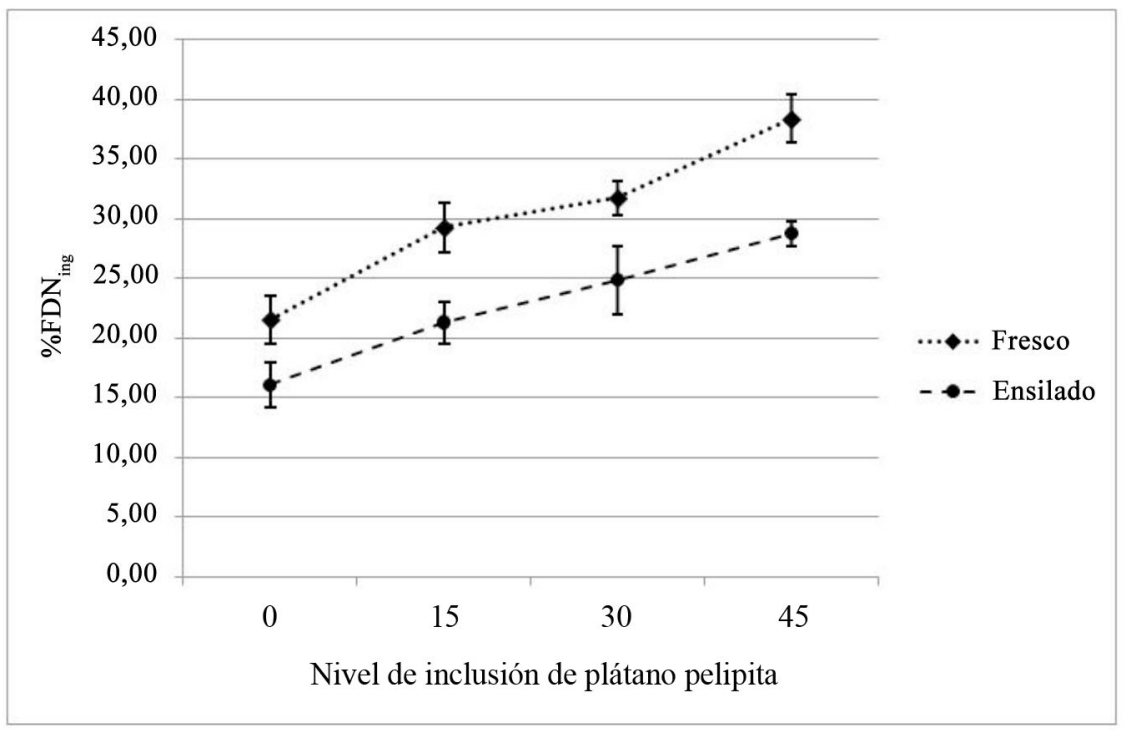

Figura 1. Concentración de fibra en detergente neutro indigestible de los tratamientos tanto en fresco, como ensilados, expresados como porcentaje del total de fibra en detergente neutro. San José, Costa Rica. 2016.

Figure 1. Concentration of indigestible neutral detergent fiber from treatments both fresh and silage, expressed as a percentage of total neutral detergent fiber. San Jose, Costa Rica. 2016. 


\section{Caracterización energética de los ensilados}

El contenido de nutrimentos digestibles totales fue influenciada por la adición del fruto de plátano, así, los tratamientos con $30 \%$ y $45 \%$ de inclusión del fruto fueron los que presentaron mayor concentración de nutrimentos digestibles, a su vez estos fueron diferentes de los tratamientos con $15 \%$ de inclusión del fruto y con adición de melaza, que fueron similares entre sí (Cuadro 5). Este aumento en el contenido de los nutrimentos digestibles provino del contenido de nutrimentos presente en el fruto de plátano Pelipita (Cuadro 1). De esta manera, aunque la fibra perdió calidad al agregar el fruto y aumentó el contenido de lignina en la mezcla, también ocurrió un aumento en el contenido de carbohidratos no fibrosos, los cuales incrementan la digestibilidad del material forrajero.

Cuadro 5. Fracciones de la energía de los ensilados de pasto Camerún (Cenchrus purpureus) con la inclusión de fruto inmaduro de plátano Pelipita (Musa ABB), posteriores a 60 días de fermentación. San José, Costa Rica. 2016.

Table 5. Energy fractions of Cameroon grass (Cenchrus purpureus) with immature Pelipita plantain fruit (Musa ABB) silage, after 60 days of fermentation. San Jose, Costa Rica. 2016.

\begin{tabular}{|c|c|c|c|c|c|}
\hline Tratamientos & $\operatorname{NDT}(\%)$ & $\mathbf{E D}^{1}$ & $\mathbf{E N}_{\mathrm{L}}$ & $\mathbf{E N}_{\text {L3x }}$ & $\mathbf{E N}_{G}$ \\
\hline pasto camerún + melaza & $56,23^{\mathrm{c}}$ & $2,48^{\mathrm{c}}$ & $1,26^{\mathrm{c}}$ & $1,26^{\mathrm{c}}$ & $0,62^{\mathrm{c}}$ \\
\hline pasto camerún $+15 \%$ plátano pelipita & $57,77^{\mathrm{c}}$ & $2,55^{\mathrm{c}}$ & $1,30^{\mathrm{c}}$ & $1,29^{\mathrm{c}}$ & $0,67^{\mathrm{c}}$ \\
\hline pasto camerún $+30 \%$ plátano pelipita & $60,52^{\mathrm{b}}$ & $2,67^{\mathrm{b}}$ & $1,36^{\mathrm{b}}$ & $1,34^{\mathrm{b}}$ & $0,75^{\mathrm{b}}$ \\
\hline pasto camerún $+45 \%$ plátano pelipita & $64,31^{\mathrm{a}}$ & $2,84^{\mathrm{a}}$ & $1,46^{\mathrm{a}}$ & $1,40^{\mathrm{a}}$ & $0,86^{\mathrm{a}}$ \\
\hline Valor de $\mathrm{p}$ & $<0,0001$ & $<0,0001$ & 0,0001 & 0,0003 & $<0,0001$ \\
\hline
\end{tabular}

${ }^{1}$ (Mcal/Kg MS)

Total de nutrimentos digestibles (TND), energía digestible (ED), energía neta de lactación $\left(\mathrm{EN}_{\mathrm{L}}\right)$, energía neta de ganancia de peso $\left(\mathrm{EN}_{\mathrm{G}}\right)$ / Total digestible nutrients (TDN), digestible energy (DE), lactation net energy $\left(\mathrm{NE}_{\mathrm{L}}\right)$, net energy for weight gain $\left(\mathrm{NE}_{\mathrm{G}}\right)$.

Letras distintas en la misma columna son diferentes $(\mathrm{p}<0,05)$ / Different letters in the same column are different $(\mathrm{p}<0.05)$.

El contenido de nutrimentos digestibles totales de los ensilados valorados fue similar que obtenidos por Cubero et al. (2010) $(57,65-66,80 \%)$ y Betancourt (2004) $(60,91 \%)$ con ensilados de maíz, además, fueron similares a los valores obtenidos por López-Herrera et al. (2009), con ensilados de rastrojos de piña con aditivos y un 15\% de pulpa de cítricos deshidratada $(59,03-66,06)$. Estas diferencias se debieron al aumento en la concentración de carbohidratos no fibrosos, aportados por el plátano, esto implicó que las mezclas ensiladas poseyesen un contenido energético superior a muchos pastos tropicales y fuesen adecuadas para ser utilizadas en raciones para rumiantes.

De la misma manera que con los nutrimentos digestibles, todas las fracciones de la energía fueron afectadas por la inclusión de plátano Pelipita; además, todos los tratamientos con adición del fruto mostraron un elevado potencial de energía para animales en producción (Cuadro 5), aún mayor que el tratamiento control. Cuando se utilizó el requerimiento energético para producir un kilogramo de leche (NRC, 2001) con un nivel de consumo de $5 \mathrm{~kg} \mathrm{MV/vaca/día,} \mathrm{los} \mathrm{materiales} \mathrm{ensilados} \mathrm{evaluados} \mathrm{mostraron} \mathrm{un} \mathrm{potencial} \mathrm{de} \mathrm{producción} \mathrm{de} \mathrm{1,5-1,7} \mathrm{kg} \mathrm{de}$ leche/vaca/día, mientras que a consumos de $15 \mathrm{~kg} \mathrm{MV/vaca/día,} \mathrm{tuvieron} \mathrm{un} \mathrm{potencial} \mathrm{para} \mathrm{producir} \mathrm{4,4-5,2} \mathrm{kg}$ de leche/vaca/día. 


\section{Literatura citada}

Abdelhadi, L.O., and F.J. Santini. 2006. Corn silage versus grain sorghum silage as a supplement to growing steers grazing high quality pastures: Effects on performance and ruminal fermentation. Anim. Feed Sci. Tech. 127:33-43.

Aganga, A.A., U.J. Omphile, T. Thema, and J.C. Baitshotlhi. 2005. Chemical composition of napier grass (Pennisetum purpureum) at different stages of growth and napier grass silages with additives. J. Biol. Sci. 5:493-496.

AOAC (Association of Official Analytical Chemist). 1998. Official Methods of Analysis of AOAC Int. 16t th $^{\text {ed., }} 4^{\text {th }}$ rev. AOAC Int., Gaithersburg, MD, USA.

Araya-Mora, M., y C. Boschini-Figueroa. 2005. Producción de forraje y calidad nutricional de variedades de Pennisetum purpureum en la Meseta Central de Costa Rica. Agron. Mesoam. 16:37-43.

Archimède, H., E. González-García, P. Despois, T. Etienne, and G. Alexandre. 2010. Substitution of corn and soybean with green banana fruits and Gliricidia sepium forage in sheep fed hay-based diets: effects on intake, digestion and growth. J. Anim. Physiol. Anim. Nutr. 94:118-128.

Archimède, H., J.L. Gourdine, A. Fanchone, R. Tournebize, M. Bassien-Capsa, and E. González-García. 2012. Integrating banana and ruminant production in the French West Indies. Trop. Anim. Health Prod. 44:1289-1296.

Barrera, J.L., G.S. Arrazola, y D.G. Cayón. 2010. Caracterización fisicoquímica y fisiológica del proceso de maduración de plátano hartón (Musa AAB Simmonds) en dos sistemas de producción. Acta Agron. 59:20-29.

Betancourt, J.C. 2004. Caracterización nutricional y productiva de material fresco y ensilado de maní forrajero (Arachis pintoi) cultivado en asocio con maíz (Zea mays), a tres densidades de siembra. Tesis MSc., Universidad de Costa Rica, San José, CRC.

Bureenok, S., C. Yuangklang, K. Vasupen, J.T. Schonewille, and Y. Kawamoto. 2012. The effects of additives in napier grass silages on chemical composition, feed intake, nutrient digestibility and rumen fermentation. Asian-Aust. J. Anim. Sci. 25:1248-1254.

Calsamiglia, S., A. Ferret, C.K. Reynolds, N.B. Kristensen, and A.M. Van Vuuren. 2010. Strategies for optimizing nitrogen use by ruminants. Animal 4:1184-1196. doi:10.1017/S1751731110000911

Cavali, J., O.G. Pereira, S.D.C. Valadares-Filho, M.O. Porto, F.E.P. Fernandes, and R. Garcia. 2010. Mixed sugarcane and elephant grass silages with or without bacterial inoculant. Rev. Bras. Zootec. 39:462-470.

Chacón-Hernández, P.A., y C.F. Vargas-Rodríguez. 2009. Digestibilidad y calidad del Pennisetum purpureum cv. king grass a tres edades de rebrote. Agron. Mesoam. 20:399-408.

Cruz-Calvo, M.M., y J.M. Sánchez. 2000. La fibra en la alimentación del ganado lechero. Nutr. Anim. Trop. 6:39-74.

Cubero, J.F., A. Rojas-Bourrillón, y R. WingChing. 2010. Uso del inóculo microbial elaborado en finca en ensilaje de maíz (Zea mays). Valor nutricional y fermentativo. Agron. Costarricense 34(2):237-250.

Detmann, E., J.T. Zervoudakis, L.D. Cabral, V.R. Rocha-Júnior, S.D. Valadares-Filho, A.C. Queiroz, N.J. Ponciano, e A.M. Fernandes. 2004. Validação de equações preditivas da fração indigestível da fibra em detergente neutro em gramíneas tropicais. Rev. Bras. Zootec. 33:1866-1875.

Di-Rienzo, J.A., F. Casanoves, M.G. Balzarini, L. Gonzalez., M. Tablada, y Y.C. Robledo. 2016. InfoStat versión 2016. Grupo InfoStat, FCA, Universidad Nacional de Córdoba, ARG. http://www.infostat.com.ar (consultado 5 ene. 2016).

Egbunuogie, B.O., and M.A. Bamikole. 2014. Nutrient content variability in three forms of elephant grass (Pennisetum purpureum) preservation. Niger. J. Agric. Food Environ. 10(1):48-52.

Espitia-Pérez, P.J., Y.J. Pardo-Plaza, y A.P. Montalvo-Puente. 2013. Características del análisis proximal de harinas obtenidas de frutos de plátanos variedades papocho y pelipita (Musa ABB Simmonds). Acta Agron. 62:189-195. 
France, J., and J. Dijkstra. 2005. Volatile fatty acid production. In: J. Dijkstra, et al., editors, Quantitative aspects of ruminant digestion and metabolism. $2^{\text {nd }}$ ed. CABI Publishing, USA. p. 157-175.

Grabber, J.H. 2005. How do lignin composition, structure, and cross-linking affect degradability? A review of cell wall model studies. Crop Sci. 45:820-831.

Gutiérrez, F., A. Rojas, H. Dormond, M. Poore, y R. WingChing. 2003. Características nutricionales y fermentativas de mezclas ensiladas de desechos de piña y avícolas. Agron. Costarricense 27(1):79-89.

Happi-Emaga, T., C. Robert, S.N. Ronkart, B. Wathelet, and M. Paquot. 2008. Dietary fibre components and pectin chemical features of peels during ripening in banana and plantain varieties. Bioresour. Technol. 99:4346-4354. doi:10.1016/j. biortech.2007.08.030

Hiriart, M. 2008. Ensilados. Procesamiento y calidad. $2^{\text {da }}$ ed. Editorial Trillas, MEX.

Hoffman, P.C. 2005. Ash content of forages. Focus on Forage 7(1):1-2.

IMN (Instituto Meteorológico Nacional). 2017. Datos climáticos Upala, IMN, CRC. https://www.imn.ac.cr/web/imn/inicio (consultado 21 feb. 2017).

Jensen, C., M.R. Weisbjerg, P. Nørgaard, and T. Hvelplund. 2005. Effect of maize silage maturity on site of starch and NDF digestion in lactating dairy cows. Anim. Feed Sci. Technol. 118:279-294.

Katongole, C.B., F.B. Bareeba, E.N. Sabiiti, and I. Ledin. 2008. Nutritional characterization of some tropical urban market crop wastes. Anim. Feed Sci. Technol. 142:275-291.

Kung, L., and R. Shaver. 2001. Interpretation and use of silage fermentation analysis reports. Focus on Frage 3(13):1-5.

Licitra, G., T.M. Hernandez, and P.J. Van-Soest. 1996. Standardization of procedures for nitrogen fractionation of ruminant feeds. Anim. Feed Sci. Technol. 57:347-358.

López-Herrera, M., y E. Briceño-Arguedas. 2016. Efecto de la frecuencia de corte y la precipitación en el rendimiento de Cratylia argentea orgánica. Nutr. Anim. Trop. 10(1):24-44.

López-Herrera, M., R. WingChing, y A. Rojas-Bourrillón. 2009. Características fermentativas y nutricionales del ensilaje de rastrojo de piña (Ananas comosus). Agron. Costarricense 33(1):1-15.

López-Herrera, M., R. WingChing-Jones, y A. Rojas-Bourrillón. 2014. Meta-análisis de los subproductos de piña (Ananas comosus) para la alimentación animal. Agron. Mesoam. 25:383-392.

López-Herrera, M., R. WingChing-Jones, y A. Rojas-Bourrillón. 2016. Bromatología de ensilados de corona de piña con pulpa de cítricos, heno y urea. Agron. Mesoam. 27:37-47.

Lounglawan, P., W. Lounglawan, and W. Suksombat. 2014. Effect of cutting interval and cutting height on yield and chemical composition of King Napier grass (Pennisetum purpureum x Pennisetum americanum). APCBEE Procedia 8:27-31.

Lu, C.D., J.R. Kawas, and O.G. Mahgoub. 2005. Fibre digestion and utilization in goats. Small Rumin. Res. 60:45-52.

Manyawu, G.J., S. Sibanda, I.C. Chakoma, C. Mutisi, and P. Ndiweni. 2003. The intake and palatability of four different types of Napier grass (Pennisetum purpureum) silage fed to sheep. Asian-Aust. J. Anim. Sci. 16:823-829.

Maza, A., G. Vergara, y D. Paternina. 2011. Evaluación química y organoléptica del ensilaje de maralfalfa (Pennisetum sp.) más yuca fresca (Manihot esculenta). Rev. MVZ Córdoba 16:2528-2537.

McDonald, P. 1981. The biochemistry of silage. John Wiley, NY, USA.

Mertens, D. 2002. Gravimetric determination of amylase-treated neutral detergent fiber in feeds with refluxing in beakers or crucibles: collaborative study. J. AOAC Int. 85:1217-1240. 
Mohapatra, D., S. Mishra, and N. Sutar. 2010. Banana and its by-product utilization: an overview. J. Sci. Ind. Res. 69:323-329.

Moore, K.J., and H.J.G. Jung. 2001. Lignin and fiber digestion. J. Range Manag. 54:420-430.

Muck, R.E., I. Filya, and F.E. Contreras-Govea. 2007. Inoculant effects on alfalfa silage: in vitro gas and volatile fatty acid production. J. Dairy Sci. 90:5115-5125.

Mühlbach, P.R. 2001. Uso de aditivos para mejorar el ensilaje de los forrajes tropicales. En: L.'t Mannetje, editor, Memorias de la conferencia electrónica de la FAO sobre el ensilaje en los trópicos: uso del ensilaje en el trópico privilegiando opciones para pequeños campesinos. FAO, Roma, ITA. p.157-171.

Muia, J.M.K., S. Tamminga, P.N. Mbugua, and J.N. Kariuki. 2000. The nutritive value of Napier grass (Pennisetum purpureum) and its potential for milk production with or without supplementation: a review. Trop. Sci. 40:109-131.

Neureiter, M., J.T.P. dos-Santos, C.P. Lopez, H. Pichler, R. Kirchmayr, and R. Braun. 2005. Effect of silage preparation on methane yields from whole crop maize silages. In: B.K. Ahring, and H. Hartmann, editors, Proceedings of the $4^{\text {th }}$ International Symposium on Anaerobic Digestion of Solid Waste. BioCentrum-DTU, Copenhagen, DEN. p. 109-115.

NRC (National Research Council). 2001. Nutrient requirements of dairy cattle. $7^{\text {th }}$ ed. National Academy Press, WA, USA.

Ramírez, R., R.G. Ramírez, y F. López. 2002. Factores estructurales de la pared celular del forraje que afectan su digestibilidad. Cienc. UANL 2:180-189.

Sampaio, C.B., E. Detmann, I. Lazzarini, M.A.D. Souza, M.F. Paulino, and S.D.C. Valadares-Filho. 2009. Rumen dynamics of neutral detergent fiber in cattle fed low-quality tropical forage and supplemented with nitrogenous compounds. Rev. Bras. Zootec. 38:560-569.

Sánchez, J.M., y H. Soto. 1998. Estimación de la calidad nutricional de los forrajes del cantón de San Carlos. II. Componentes de la pared celular. Nutr. Anim. Trop. 4(1):7-19.

Thomas, M.E., J.L. Foster, K.C. McCuistion, L.A. Redmon, and R.W. Jessup. 2013. Nutritive value, fermentation characteristics, and in situ disappearance kinetics of sorghum silage treated with inoculants. J. Dairy Sci. 96:7120-7131.

Tobia, C. 2004. Introducción del ensilaje de soya en un sistema de producción intensiva de leche en el trópico húmedo de Costa Rica. Tesis Doc., Universidad de Costa Rica, San José, CRC.

Van-Soest, PJ. 1994. Nutritional ecology of the ruminant. $2^{\text {th }}$ ed. Cornell Univesity Press, NY, USA.

Van-Soest, P. J., M.E. Van Amburgh, J.B. Robertson, and W.F. Knaus. 2005. Validation of the 2.4 times lignin factor for ultimate extent of NDF digestion, and curve peeling rate of fermentation curves into pools. In: Cornell University, editor, Proceedings of the Cornell Nutritional Conference for Feed Manufacturers. Cornell University, Ithaca, NY, USA. p. 139-149.

Ward, J.D., D.D. Readfern, M.E. McCormick., and G.J. Cuomo. 2001. Chemical composition, ensiling characteristics, and apparent digestibility of summer annual forages in a subtropical double-cropping system with annual ryegrass. J. Dairy Sci. 84:177-182.

Weiss, W.P. 2004. Fine-tuning energy calculations. In: M. Eastridge, editor, Proceedings of the Tri-State Dairy Nutrition Conference. Purdue University, Michigan State University and Ohio State University, Fort Wayne, IN, USA. p. 131-142.

Yang, H.Y., X.F. Wang, J.B. Liu, L.J. Gao, M. Ishii, Y. Igarashi, and Z.J. Cui. 2006. Effects of water-soluble carbohydrate content on silage fermentation of wheat straw. J. Biosci. Bioeng. 101:232-237.

Yitbarek, M.B., and B. Tamir. 2014. Silage additives: review. Open J. Appl. Sci. 4:258-274.

Zanine, A.D.M., E.M. Santos, J.R.R Dórea, P.A.D.S. Dantas, T.C.D. Silva, and O.G. Pereira. 2010. Evaluation of elephant grass silage with the addition of cassava scrapings. Rev. Bras. Zootec. 39:2611-2616. 